\title{
Valence band hard x-ray photoelectron spectroscopy on $3 d$ transition-metal oxides containing rare-earth elements
}

\author{
D. Takegami, ${ }^{1}$ L. Nicolai, ${ }^{2}$ T. C. Koethe, ${ }^{3}$ D. Kasinathan, ${ }^{1}$ C. Y. Kuo,${ }^{1,4}$ Y. F. Liao, ${ }^{4}$ K. D. Tsuei, ${ }^{4}$ G. Panaccione,${ }^{5}$ \\ F. Offi, ${ }^{6}$ G. Monaco, ${ }^{7}{ }^{*}$ N. B. Brookes, ${ }^{7}$ J. Minár, ${ }^{2}$ and L. H. Tjeng ${ }^{1}$ \\ ${ }^{1}$ Max Planck Institute for Chemical Physics of Solids, Nöthnitzer Straße 40, 01187 Dresden, Germany \\ ${ }^{2}$ New Technologies-Research Center, University of West Bohemia, Univerzitni 8, 30614 Pilsen, Czech Republic \\ ${ }^{3}$ Institute of Physics II, University of Cologne, Zülpicher Straße 77, 50937 Cologne, Germany \\ ${ }^{4}$ National Synchrotron Radiation Research Center (NSRRC), 101 Hsin-Ann Road, 30076 Hsinchu, Taiwan \\ ${ }^{5}$ TASC Laboratory, IOM-CNR, Area Science Park, S.S.14, Km 163.5, I-34149 Trieste, Italy \\ ${ }^{6}$ CNISM and Dipartimento di Scienze, Università Roma Tre, Via della Vasca Navale 84, I-00146 Rome, Italy \\ ${ }^{7}$ European Synchrotron Radiation Facility, BP220, 38043 Grenoble, France
}

(Received 14 December 2018; revised manuscript received 15 February 2019; published 2 April 2019)

\begin{abstract}
Here we report on our study to quantitatively describe the intensities of the valence band hard x-ray photoemission spectra (HAXPES) of a rare earth element containing $3 d$ transition metal oxides. Using $\mathrm{LaCoO}_{3}$ as a representative model compound, we compared the experimental data to the results of $a b$ initio one-step photoemission band structure calculations as well as to the sum of the partial density of states of the atomic constituents weighted by their tabulated photoionization cross sections. We discovered that the semicore La $5 p$ density of states surprisingly contributes in a significant manner to the valence band spectrum: Although the La $5 p$ partial density of states in the valence band region is negligible compared to that of the $\mathrm{O} 2 p$ or the Co $3 d$, the La $5 p$ cross section in the hard $\mathrm{x}$-ray range is found to be orders of magnitude larger than that of the other subshells. This explains the long-standing issue of why the hard x-ray valence band spectra of a rare-earth element containing materials have line shapes that are very different from those taken at lower photon energies and why they cannot be described in terms of partial density of states of the subshells usually considered for the lower photon energy spectra. We infer that the contribution of the rare-earth $5 p$ must be taken into account and cannot be ignored.
\end{abstract}

DOI: 10.1103/PhysRevB.99.165101

\section{INTRODUCTION}

Hard x-ray photoelectron spectroscopy (HAXPES) has been recently established as a mature technique for investigating the bulk electronic structure of solids [1]. In addition to the direct advantages coming from the high photon energies such as the higher probing depth or the possibility of analyzing deeper core levels, it has also proved to have further benefits with respect to its lower energy counterparts, such as an increased sensitivity to $s$ and $p$ orbitals or the photoelectron angular distribution when using linear polarized light. These characteristics allowed the identification of the character of the orbitals contributing to the valence band in a very wide range of materials [2-12].

Nonetheless, it is somewhat surprising that the number of valence band HAXPES studies on especially rare earth (e.g., La) containing $3 d$ transition metal oxides is so far

*Present address: Department of Physics, University of Trento, Via Sommarive, 14-38123 Povo, Italy.

Published by the American Physical Society under the terms of the Creative Commons Attribution 4.0 International license. Further distribution of this work must maintain attribution to the author(s) and the published article's title, journal citation, and DOI. rather limited, despite the fact that there is a strong need for bulk sensitive valence band spectra of this important class of materials which show spectacular quantum phenomena such as high $T_{c}$ superconductivity, colossal magnetoresistance, metal-insulator and spin-state transitions [13,14]. Part of the problem is related to the very small photoionization cross sections of the transition metal $3 d$ electrons for hard $\mathrm{x}$ rays [15-18]. Here we would like to argue, however, that also a quantitative interpretation of the spectra is actually very challenging. This is not only because of the presence of strong electron correlations but also because the spectra cannot be interpreted in terms of a weighted sum of the transition metal $3 d$, oxygen $2 p$, and/or rare-earth $4 f$ spectral weights as usually performed for valence band spectra taken with ultraviolet or soft $\mathrm{x}$-ray photon energies.

To demonstrate our point, we have carried out a quantitative investigation on the HAXPES valence band spectrum of $\mathrm{LaCoO}_{3}$ as a representative for the class of rare earth containing $3 d$ transition metal oxides. $\mathrm{LaCoO}_{3}$ is a nonmagnetic insulator at low temperatures, with the $\mathrm{Co}^{3+} 3 d^{6}$ ion in the octahedral oxygen coordination having the low spin $t_{2 g}^{6}$ configuration [19-25]. Despite the presence of strong electronic correlations, nonmagnetic $\mathrm{LaCoO}_{3}$ with a completely filled $t_{2 g}$ and empty $e_{g}$ can effectively be considered as a band insulator, for which ab initio band structure calculations can be expected to provide a reasonable description of its 
electronic structure. This is important since we will make use of one-step photoemission calculations [1,26-28] based on such band structure results to explain the valence band HAXPES spectrum and to study whether the sum of the partial density of states of the atomic constituents weighted by their tabulated photoionization cross sections can reproduce the experiment.

\section{METHODS}

The HAXPES experiments have been carried out at the Max-Planck-NSRRC end station at the Taiwan undulator beamline BL12XU at SPring-8, Japan. The photon beam was linearly polarized with the electrical field vector in the plane of the storage ring (i.e., horizontal) and the photon energy was set at about $6.5 \mathrm{keV}$. The experimental setup has two MB Scientific A-1 HE analyzers, see Fig. 1. In the so-called parallel geometry, we used the analyzer which was mounted horizontally and parallel to the photon beam's electrical field vector $\theta=0^{\circ}$, where $\theta$ is the angle between the electrical field and the momentum of the photoelectron). In the perpendicular geometry, we used the analyzer which was mounted vertically and perpendicular to the electrical field vector $\left(\theta=90^{\circ}\right)$ and the Poynting vector of the beam. With this setup we can perform polarization dependent experiments with the full light polarization provided by the undulator beam line, without the loss of intensity that otherwise would occur when using phase retarders. The overall energy resolution was set at $0.27 \mathrm{eV}$. The measurements were performed at $80 \mathrm{~K}$.

Soft x-ray photoelectron spectroscopy (SXPS) was also performed. This was carried out at the ID8 beamline of the ESRF equipped with a Scienta SES-2002 electron energy analyzer. The analyzer is mounted in the horizontal plane at a $60^{\circ}$ angle relative to the incident light. The photon energy was set at $700 \mathrm{eV}$ and the light polarization was linear in the horizontal plane. The overall energy resolution was $0.2 \mathrm{eV}$. The measurement temperature was $65 \mathrm{~K}$.

Single crystals of $0.2 \%$ and $1 \% \mathrm{Sr}$ doped $\mathrm{LaCoO}_{3}$ have been grown by the traveling floating-zone method in an image furnace. The $0.2 \% \mathrm{Sr}$ sample was used for the SXPS and the $1 \%$ for HAXPES in order to avoid charging problems that may otherwise occur associated with the insulating character of $\mathrm{LaCoO}_{3}$ at low temperatures. Magnetic susceptibility measurements were performed with a Quantum Design's MPMS 3 , verifying that the curves reproduce data reported earlier $[29,30]$. The single crystals were cleaved in situ in the ultrahigh vacuum experimental setups in order to obtain a clean surface.

The $a b$ initio calculations presented in this work were performed using the SPR-KKR package [28]. The package is a Green's function formalism based code relying on the multiple scattering theory using the Korringa-Kohn-Rostoker method. The SPR-KKR is founded on the fully relativistic Dirac equation, thus implying inclusion of all relativistic effects. The ground state properties of $\mathrm{LaCoO}_{3}$ were derived from density functional theory (DFT) calculations carried out within the local density approximation (LDA). The theoretical photoemission spectra were obtained using the one-step model of photoemission as included in the SPR-KKR package $[26,27]$. The angle-integrated photoemission spectroscopy

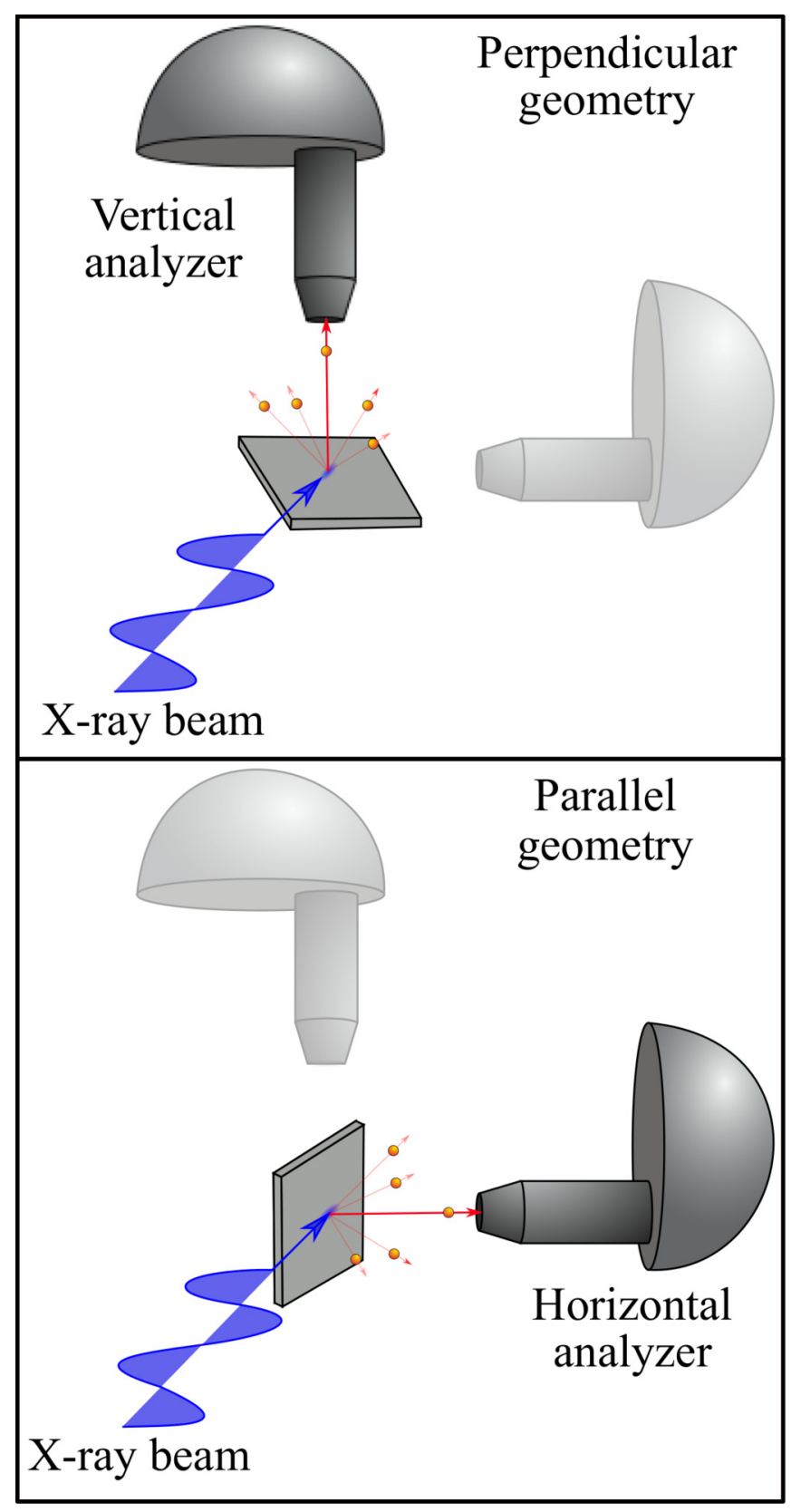

FIG. 1. Experimental geometry of the HAXPES experiment. Top panel: In the perpendicular geometry, the outgoing photoelectrons detected by the vertically mounted analyzer have their momentum perpendicular to the electrical field of the photon beam. Bottom panel: In the horizontal geometry, the photoelectrons detected by the horizontally mounted analyzer have their momentum parallel to the electrical field of the photon beam. The electrical field is always horizontal, in the plane of the synchrotron storage ring.

(AIPES) results from an integration of the initial state over the Brillouin zone. The kinematics, e.g., $\mathrm{k}$ dependence of the final state is still taken into account [27]. This way the multiple scattering effects of the final state (e.g., photoelectron diffraction related intensity variations), which are discussed in Sec. III on the atomic cross sections, are considered. AIPES calculations are then performed in a certain angular window, which corresponds to the geometry and slit of the detector, and 

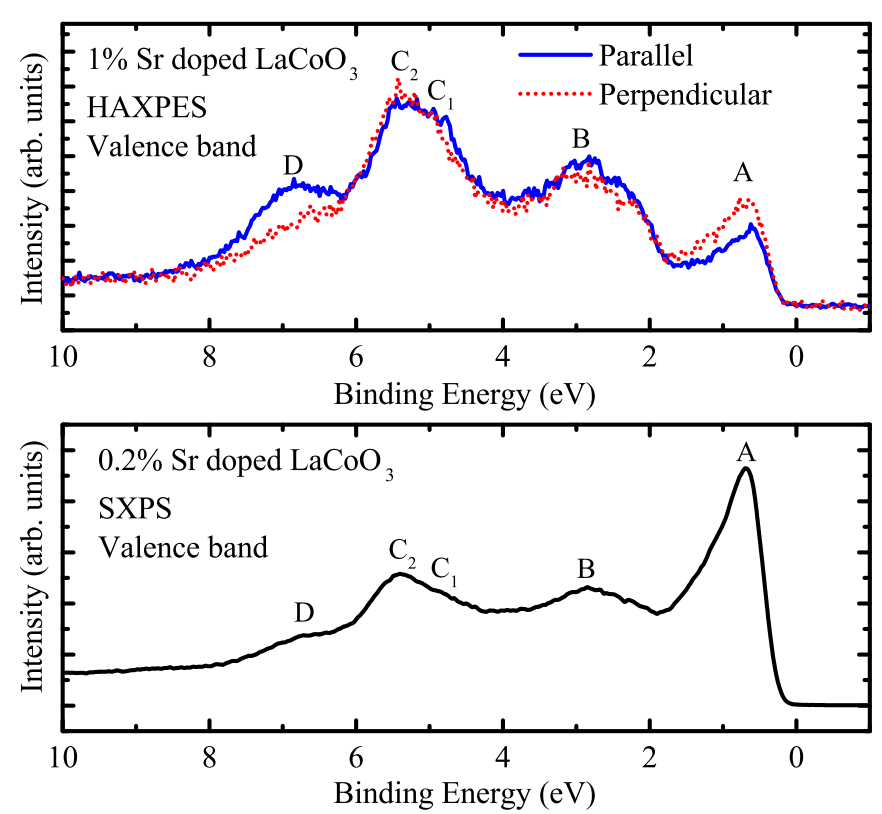

FIG. 2. Top panel: Valence band HAXPES spectra $(h v=$ $6.5 \mathrm{keV})$ of the $1 \% \mathrm{Sr}$ doped $\mathrm{LaCoO}_{3}$ taken in the parallel $(\theta=$ $\left.0^{\circ}\right)$ and vertical $\left(\theta=90^{\circ}\right)$ geometries. Bottom panel: Valence band SXPS spectrum $(h v=700 \mathrm{eV})$ of $0.2 \% \mathrm{Sr}$ doped $\mathrm{LaCoO}_{3}$.

consequently the $\mathrm{k}$ dependence of the final state is integrated out. It is also important to notice that all photoemission calculations have been performed for the experimental geometry shown in Fig. 1 using the dipole approximation. This guarantees that all dichroic effects due to the relative arrangement of the light polarization and the detector are taken into account. The photon energy used for all presented AIPES spectra was $6.5 \mathrm{keV}$.

\section{EXPERIMENTAL RESULTS}

The top panel of Fig. 2 shows the HAXPES valence band spectra taken with the parallel (blue line) and perpendicular (red dots) geometries. The intensities of the parallel vs perpendicular spectra were normalized by their integrated intensity. For comparison, we plot in the bottom panel of Fig. 2 the SXPS spectrum. In the spectra, four main features can be observed, labeled as A, B, C (1 and 2), and D. In the literature, based mostly on spectra using ultraviolet or soft x-ray light ( $h v \leqslant 1.5 \mathrm{keV}$ ), the peak closest to the zero binding energy, $\mathrm{A}$, is usually attributed mostly to the Co $3 d$, while the features $\mathrm{B}, \mathrm{C}$, and $\mathrm{D}$ are assigned more to the oxygen $2 p$ [31]. One of the main differences between the HAXPES and SXPS spectra is that in HAXPES peak A is much smaller than the features B and C while in the SXPS the situation is completely opposite. This is unexpected if one considers the photon energy dependence of the photoionization cross section of the Co $3 d$ versus that of the $\mathrm{O} 2 p$. According to the tabulated values in the literature [15-18], the ratio of the Co $3 d$ to $\mathrm{O}$ $2 p$ cross sections is about 7.5 at $6.5 \mathrm{keV}$ and about 7.2 at $700 \mathrm{eV}$, see Table I. Thus the ratio between peak A vs features $\mathrm{B}$ and $\mathrm{C}$ should not change much when comparing HAXPES with SXPS. The experiment, however, is very different and this discrepancy clearly requires explanation. Also the
TABLE I. Subshell photoionization cross section $(\sigma)$ at $6.5 \mathrm{keV}$ and $700 \mathrm{eV}$ deduced from Refs. [16-18]. $\sigma$ is divided by the number of electrons in the subshell. $\beta$ denotes the dipole parameter of the angular distribution. The cross sections for parallel and perpendicular geometries are obtained by $\sigma[1+\beta(1 / 4+3 / 4 \cos (2 \theta))]$, with $\theta=$ $0^{\circ}$ and $\theta=90^{\circ}$, respectively.

\begin{tabular}{|c|c|c|c|c|c|}
\hline \multicolumn{5}{|c|}{$h v=6.5 \mathrm{keV}$} & \multirow{2}{*}{$\begin{array}{c}h v=700 \mathrm{eV} \\
\sigma / \mathrm{e}^{-} \\
(\mathrm{kb})\end{array}$} \\
\hline $\begin{array}{l}\text { Atomic } \\
\text { subshell }\end{array}$ & $\begin{array}{c}\sigma / \mathrm{e}^{-} \\
\left(10^{-3} \mathrm{~kb}\right)\end{array}$ & $\beta$ & $\begin{array}{c}\text { Parallel } \\
\left(10^{-3} \mathrm{~kb}\right)\end{array}$ & $\begin{array}{c}\text { Perp. } \\
\left(10^{-3} \mathrm{~kb}\right)\end{array}$ & \\
\hline $\operatorname{La} 5 p_{1 / 2}$ & 109 & 1.48 & 270 & 28.3 & 5.59 \\
\hline $\mathrm{La} 5 p_{3 / 2}$ & 96 & 1.56 & 246 & 21.1 & 6.12 \\
\hline $\mathrm{La} 6 s_{1 / 2}$ & 9.75 & 1.99 & 29.1 & 0.06 & 0.74 \\
\hline $\mathrm{La} 5 d_{3 / 2}$ & 8.33 & 0.95 & 16.3 & 4.36 & 4.01 \\
\hline $\operatorname{Co} 4 s_{1 / 2}$ & 21.0 & 1.98 & 62.6 & 0.18 & 1.79 \\
\hline $\operatorname{Co} 3 d_{3 / 2}$ & 1.53 & 0.34 & 2.06 & 1.27 & 10.5 \\
\hline Co $3 d_{5 / 2}$ & 1.47 & 0.36 & 2.00 & 1.21 & 10.3 \\
\hline O $2 p_{1 / 2}$ & 0.20 & 0.09 & 0.22 & 0.19 & 1.43 \\
\hline $\mathrm{O} 2 p_{3 / 2}$ & 0.20 & 0.10 & 0.22 & 0.19 & 1.42 \\
\hline
\end{tabular}

polarization dependence in the HAXPES experiment is seemingly inconsistent with the tabulated photoionization crosssection values as we will show next. Table I lists these values at $h v=6.5 \mathrm{keV}$ as interpolated from the numbers in the literature [16-18]. The values after including the effect of the angular dependence at the parallel and perpendicular geometries have been calculated by using the following equation [16-18]:

$$
\left.\frac{d \sigma}{d \Omega}=\frac{\sigma}{4 \pi}\left[1+\beta P_{2} \cos \theta+\left(\gamma \cos ^{2} \theta+\delta\right) \sin \theta \cos \varphi\right)\right],
$$

where $\sigma$ is the subshell photoionization cross section and $P_{2}$ the second Legendre polynomial. $\varphi$ is here the angle between the photon momentum vector and the projection of the photoelectron momentum vector on the plane perpendicular to the electrical field vector and containing the photon momentum vector. The first two summands in Eq. (1) represent a dipolar approximation; the third summand includes nondipolar effects. We have for the parallel geometry, i.e., with the analyzer along the electric field vector, $\theta=0^{\circ}$ ( $\varphi$ undefined) and for the perpendicular geometry $\theta=90^{\circ}$ and $\varphi=90^{\circ}$. The expression Eq. (1) now simplifies to

$$
\frac{d \sigma}{d \Omega}=\frac{\sigma}{4 \pi}\left[1+\beta\left(\frac{1}{4}+\frac{3}{4} \cos 2 \theta\right)\right] .
$$

Comparing in Fig. 2(a) the spectra obtained using the two geometries, we observe that the main differences are the suppression of feature D and the increase of intensity of peak $\mathrm{A}$ in the perpendicular geometry with respect to the parallel one (the two spectra were normalized to their integrated intensity). If we assume that part of feature D is due to a Co $4 s$ contribution, in analogy to the appearance of the $\mathrm{Ni} 4 s$ reported for HAXPES measurements on $\mathrm{NiO}$ [7], the cross sections (see Table I) would indeed justify its suppression: There is a decrease by two orders of magnitude for the Co $4 s$ cross section in going from the parallel to the perpendicular geometry. Yet, if we assume that a large part of features $\mathrm{B}$ and $\mathrm{C}$ is due to the $\mathrm{O} 2 p$ and that peak $\mathrm{A}$ is given 


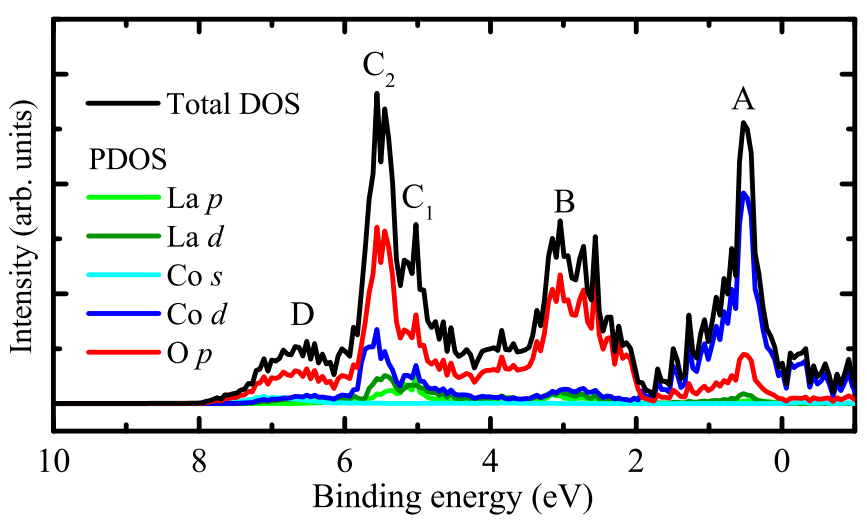

FIG. 3. Calculated density of states (DOS) of $\mathrm{LaCoO}_{3}$ and partial density of states (PDOS) of the orbitals relevant to our study. The contribution of the La $s$ PDOS is not plotted since it is even much smaller than the Cos.

by the Co $3 d$, then the cross sections (Table I) would predict a decrease of peak A relative to features $\mathrm{B}$ and $\mathrm{C}$ in going from parallel to perpendicular geometry. The experiment shows the opposite trend. These observations lead us to conclude that the HAXPES valence band spectra cannot be explained in terms of Co $3 d$ and $\mathrm{O} 2 p$ spectral weights only.

\section{CALCULATIONS AND ANALYSIS}

In order to identify the origin of the valence band features observed in HAXPES, we carried out LDA calculations for $\mathrm{LaCoO}_{3}$ in the nonmagnetic phase. The total density of states (DOS) and the partial density of states (PDOS) are displayed in Fig. 3. We can observe that peak A is mostly due to the Co $d$ and that features B-C1-C2-D are originating primarily from the $\mathrm{O} p$, consistent with earlier analyses in the literature [31]. For completeness, we also show in Fig. 3 the PDOS of the Co $s$, the La $p$, and La $d$. They are tiny, but we will show below that they are important for the interpretation of the HAXPES signal. We have not plotted the contribution of the La $s$ PDOS since it is even much smaller than the Cos [32].

In order to compare the LDA calculations with our HAXPES and SXPS spectra, we scaled the PDOS by their respective photoionization cross sections as shown in Table I. We then multiplied them with the Fermi function, applied a broadening using a Gaussian of $0.25 \mathrm{eV}$ (HAXPES) or $0.2 \mathrm{eV}$ (SXPS) full width at half maximum (FWHM) and a Lorentzian of $0.1 \mathrm{eV}$ FWHM, to account for experimental resolution and lifetime, respectively, and calculated their sum. We also have carried out calculations with the onestep method to directly simulate the HAXPES valence band spectra.

In Fig. 4 we show the results for the SXPS spectrum. We can observe that the sum of the PDOS weighted by the respective photoionization cross sections at $700 \mathrm{eV}$ photon energy reproduces quite well the overall experimental spectrum, in particular that peak A is more intense than features B-C-D. We identify the Co $3 d$ and the $\mathrm{O} 2 p$ as the major contributors to the SXPS spectrum, thereby confirming the earlier assignment in the literature [31]. For SXPS, we can safely neglect the contribution of the Co $4 s$, La 5p, La 5d, and La $6 s$ PDOS.

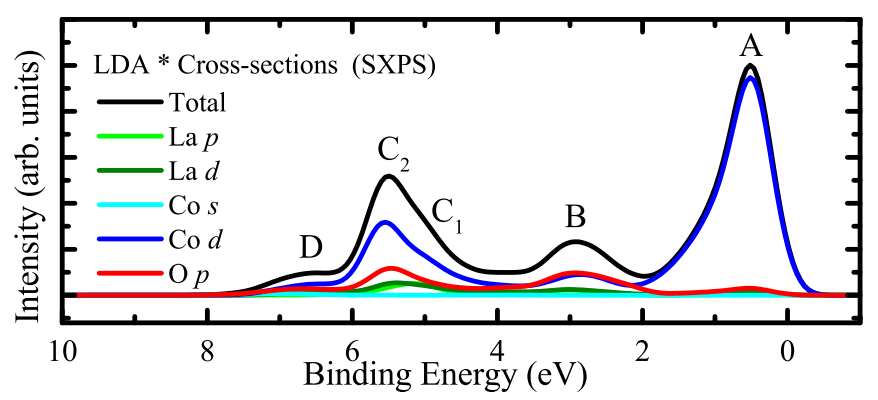

FIG. 4. Simulated valence band SXPS spectrum of $\mathrm{LaCoO}_{3}$ obtained by multiplying the $\operatorname{Co} d$, O $p$, Co $s$, La $p$, La $s$, and La $d$ PDOS from the LDA calculations by their respective photoionization cross section at $700 \mathrm{eV}$ photon energy, followed by their summation. The contribution of the La $s$ PDOS is not plotted since it is even much smaller than the Co $s$.

Figure 5 displays the experimental HAXPES spectra together with the simulations for the HAXPES spectra using the cross-section analysis and the one-step calculations. We will start with the results for the parallel geometry, Fig. 5(a). There we can observe a good agreement between the sum of the PDOS weighted by the respective photoionization cross sections at $6.5 \mathrm{keV}$ photon energy (middle panel) and the experimental spectrum (top panel). Unlike in SXPS, the simulations show that peak $\mathrm{A}$ is not the strongest feature in HAXPES spectrum, but rather features $\mathrm{B}-\mathrm{C} 1-\mathrm{C} 2$. The reason for this is the very large relative contribution of the La $5 p$. This comes as a surprise since the weight of the La $5 p$ PDOS is one order of magnitude smaller in comparison to that of the Co $3 d$ or $\mathrm{O} 2 p$ as shown in Fig. 3. However, the photoionization cross section of La $5 p$ is about two orders larger than that of the Co $3 d$ or O $2 p$, see Table I. So in HAXPES we apparently are most sensitive to the tiny La $5 p$ PDOS that are mixed into the valence band made of Co $3 d$ and O $2 p$ states.

Since the above mentioned analysis depends on small numbers (La $5 p$ PDOS) multiplied by large numbers (La $5 p$ cross section), we need an independent verification for which the one-step method comes into play. The bottom panel of Fig. 5(a) shows the photoemission spectrum as calculated by this one-step method from the LDA results for $\mathrm{LaCoO}_{3}$. We can clearly observe that the agreement with the experiment (top panel) is excellent. All spectral features are well reproduced in terms of energy positions and intensities. This supports the notion that indeed the La $5 p$ PDOS is contributing strongly to the HAXPES signal despite its very small weight in the valence band. We would like to note that the one-step calculation reproduces the intensity of peak D much better than the cross-section analysis. At the moment we can only speculate that the inaccuracies in the cross-section analysis may originate from the fact that the assignments in terms of the various orbital PDOS may not be unique and rather depends on the wave-functions basis set used in the band structure calculation and that the orbitals are thus also different from the atomic ones used in the calculations for the photoionization cross sections.

Next we will investigate the results for the perpendicular geometry. We can observe from Fig. 5(b) that also here there is a reasonable overall agreement between the sum of the PDOS 
(a) Parallel

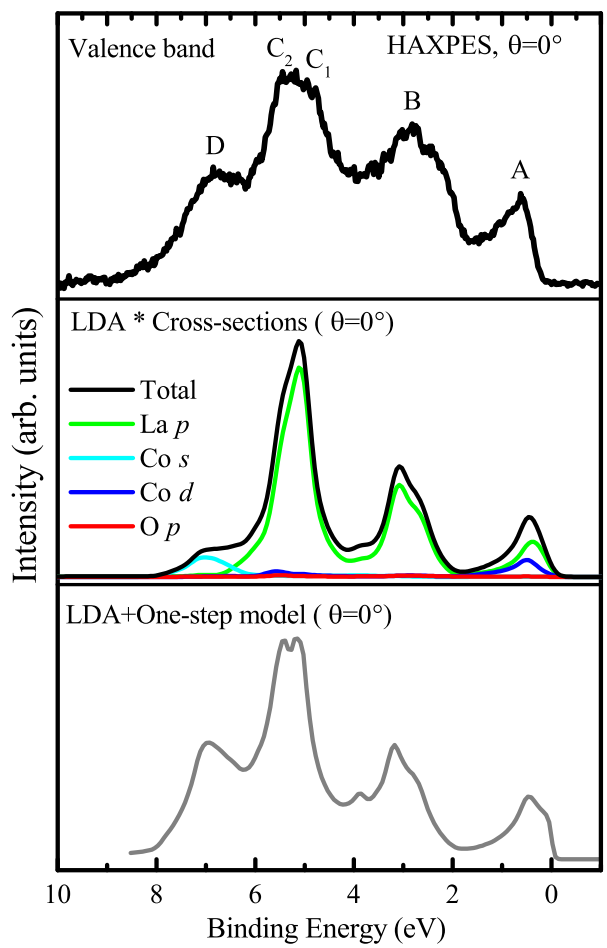

(b) Perpendicular

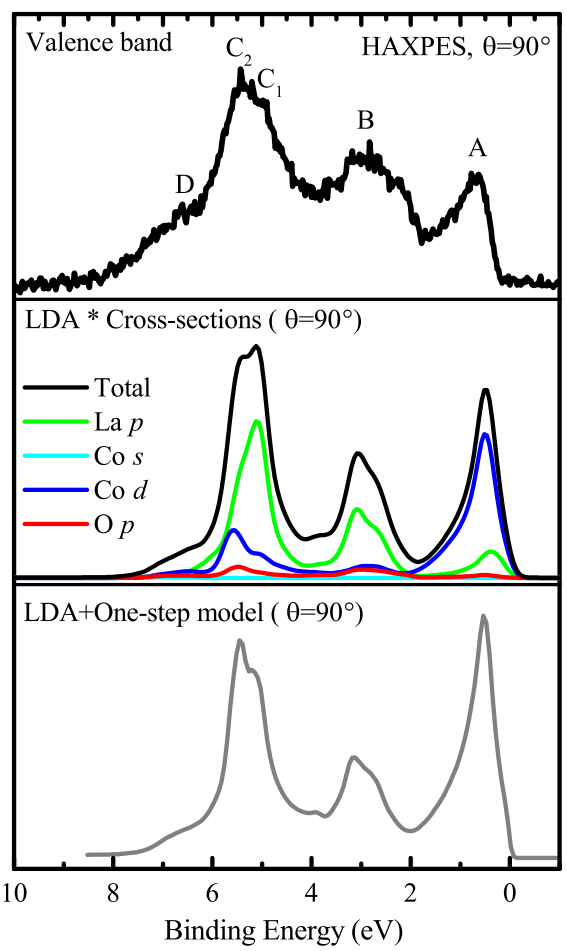

(c) Perpendicular (effective angular acceptance)
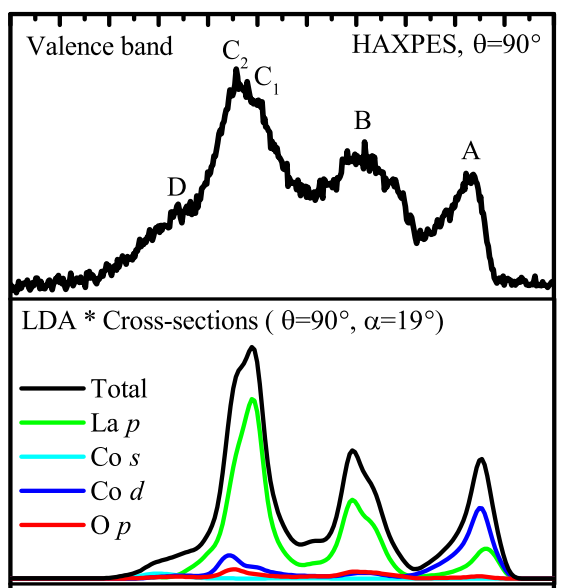

LDA+One-step model $\left(\theta=90^{\circ}, \alpha=19^{\circ}\right)$

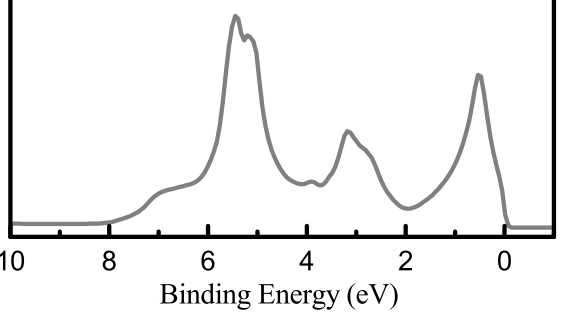

FIG. 5. Experimental HAXPES valence band spectra (upper panels) compared with the simulated spectra obtained by multiplying the Co $d$, O $p$, Co $s$, La $p$, La $s$, and La $d$ PDOS from the LDA calculations by their respective photoionization cross section (middle panels) followed by their summation and the results after using the one-step model on the LDA calculations (lower panels). Panels (a) and (b) show the results corresponding to the parallel $\left(\theta=0^{\circ}\right)$ and perpendicular $\left(\theta=90^{\circ}\right)$ geometry, respectively. Panel (c) shows the results for the perpendicular geometry after including the effective angular acceptance as described in the text and in Ref. [7]. For the one-step model results, the correction was taken into account by numerically integrating spectra calculated at several angles.

weighted by the respective cross sections (middle panel) and the experimental spectrum (top panel). The same observation can also be made for the spectrum calculated using the onestep model (bottom panel). In comparing the perpendicular geometry with the parallel geometry, the lower intensity of feature $\mathrm{D}$ in the experiment is reproduced by the simulations. This confirms that the Co $4 s$ indeed contributes to the intensity of feature $\mathrm{D}$ in the parallel geometry but is practically forbidden in the perpendicular geometry, see Table I. The other important change, namely the increase of the intensity of peak A relative to that of features B-C1-C2 in going from parallel to perpendicular geometry, is also reproduced in the simulations. Table I shows the reason why: The La $5 p$ has a considerably higher $\beta$-asymmetry parameter value than the Co $3 d$, meaning that the suppression of the signal in going from parallel to perpendicular geometry is stronger for the La $5 p$ (factor 10 reduction) than for the Co $3 d$ (factor two reduction). The fact that this lineshape change is highly visible in HAXPES supports again the notion that the La $5 p$ is indeed important for the HAXPES signal.

A closer look at the simulations for the perpendicular geometry reveals, however, that the calculated intensity ratio of peak A vs features B-C-D is too large in comparison to the experimental one. This is the case for both the crosssection analysis and the one-step method calculations. The problem is perhaps not so much that peak A is too large in the simulations but rather that features B-C-D are too small. The experiment apparently does not show the expected factor of 10 reduction for the La $5 p$. A similar phenomenon has been previously reported [7]: In studying $\mathrm{NiO}$, it was noted that the suppression of the $\mathrm{O} 1 s$ core level and $\mathrm{Ni} 4 s$ valence band intensity in going from the parallel to perpendicular geometry was very strong but not as complete as expected on the basis of the $\beta$ parameter being very close to 2 . This incomplete suppression was attributed to $\mathrm{O} 1 s$ and valence band photoelectrons quasielastically scattered while traveling in the solid and yet arriving at the analyzer although they had a different direction when they were created by the photoelectric effect. It turned out that an effective angular acceptance of the photoelectrons of about $\pm 18^{\circ}$ was able to describe this phenomenon quantitatively [7].

We now will determine the numbers for our $\mathrm{LaCoO}_{3}$ case. Figure 6 displays the HAXPES Co $2 p$ and $\mathrm{O} 1 s$ core level spectra. The parallel vs perpendicular spectra are normalized with respect to the Co $2 p$ main peak intensity. Also here it is clear that the suppression of the $\mathrm{O} 1 s$ in the perpendicular geometry is not complete. The tabulated angular dependence of the $\mathrm{O} 1 s$ at $h v=6.5 \mathrm{keV}$ [16-18] is plotted in Fig. 7 and gives clearly zero intensity for the perpendicular geometry.

To reproduce the experimentally observed finite intensity, we have to assign an effective angular acceptance $\pm \alpha$ of about 


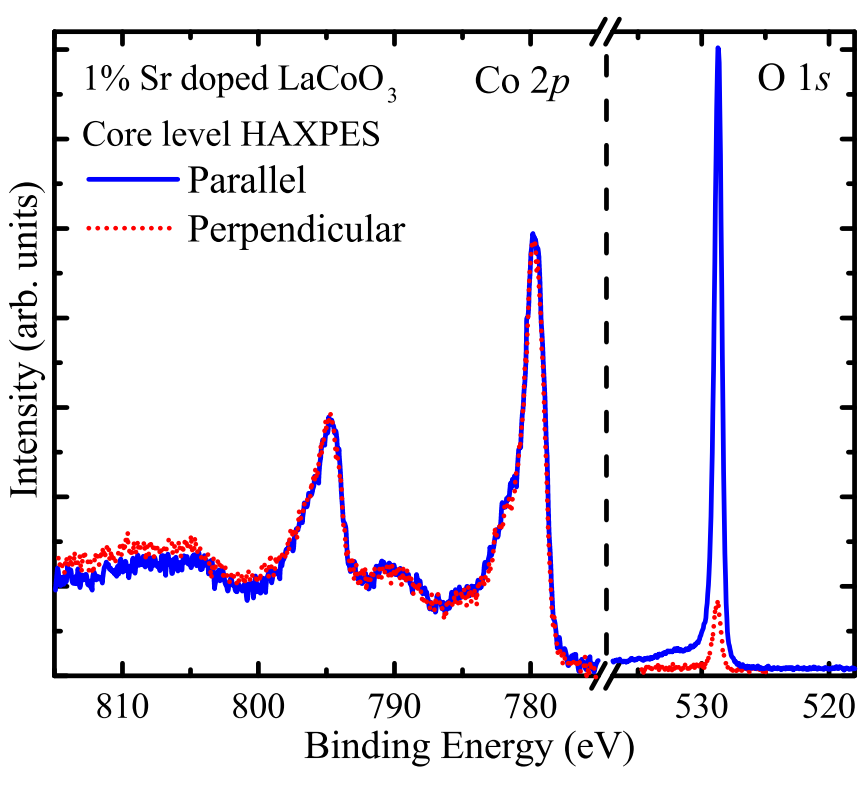

FIG. 6. Co $2 p$ and $\mathrm{O} 1 s$ core level HAXPES spectra of the $1 \% \mathrm{Sr}$ doped $\mathrm{LaCoO}_{3}$ with $\theta=0^{\circ}$ (parallel) and $\theta=90^{\circ}$ (perpendicular). The spectra has been normalized to the Co $2 p$ core level peak intensities.

$\pm 19^{\circ}$ to account for the presence of quasielastic scattering processes, i.e., we have to integrate the signal from $\theta=(90-$ $\alpha)^{\circ}$ to $\theta=(90+\alpha)^{\circ}$ (followed by a normalization of $2 \cdot \alpha$ with $\alpha$ in rad) as illustrated by the gray shaded angular fan in Fig. 7. We note that this $\pm 19^{\circ}$ number is very similar as in the $\mathrm{NiO}$ case [7]. We also would like to point out that the consideration for quasielastic scattering effects is important for the perpendicular geometry but much less so for the

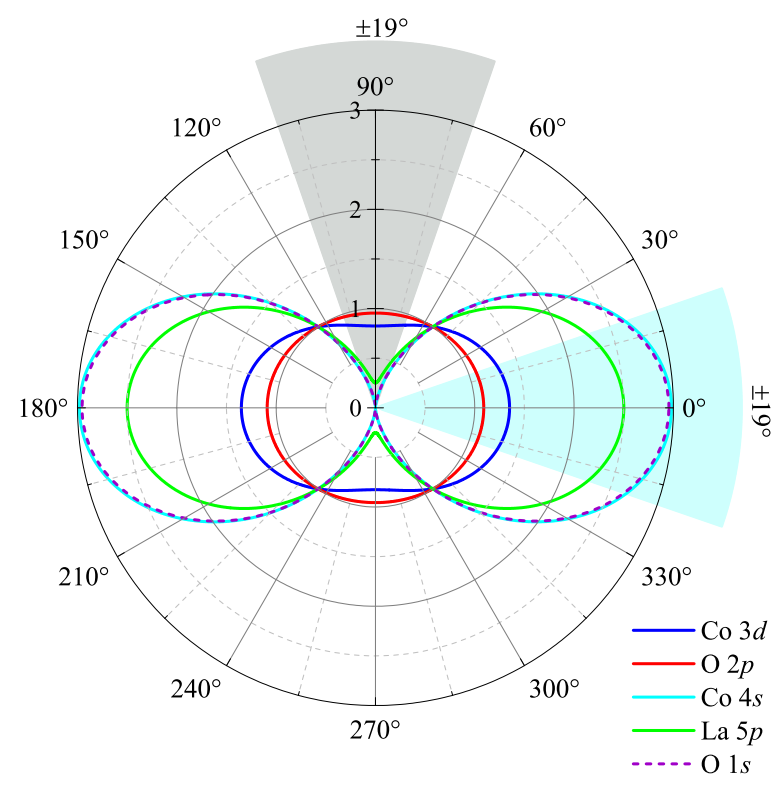

FIG. 7. Polar plot of the cross section angular dependence of the Co $3 d$, O $2 p$, Co $4 s$, La $5 p$, and O $1 s$ orbitals at $h v=6.5 \mathrm{keV}$ interpolated from Refs. [16-18]. The shaded angular fans indicate the effective angular acceptance as a result of quasielastic scattering processes. parallel geometry since the relative variation of the intensity with angle is much larger in the perpendicular than in the horizontal geometry, i.e., compare the intensity variation in the gray shaded with that in the blue shaded angular fans in Fig. 7.

We now apply this $\pm 19^{\circ}$ angular acceptance also for the valence band simulation. The results are shown in the middle panel of Fig. 5(c). For the one-step calculation, the correction was achieved by calculating the spectra at $\theta \in\left\{90^{\circ},(90 \pm\right.$ $\left.9.5)^{\circ},(90 \pm 19)^{\circ}\right\}$ followed by an interpolative numerical integration. The results are shown in the bottom panel of Fig. 5(c). We can observe that we are now able to achieve a very good quantitative agreement to the experimental data, which proves that our approach is physically justified and thus verifies our conclusions.

\section{CONCLUSIONS}

We have carried out a detailed HAXPES valence band study on $\mathrm{LaCoO}_{3}$. We were able to disentangle the various orbital contributions to the spectra using a combination of band structure calculations, photoionization cross-section analysis, and the one-step photoemission method. We discovered that the semicore La $5 p$ contributes significantly to the HAXPES valence band spectra. This is to be contrasted with valence band spectra taken with soft $\mathrm{x}$ rays, where the Co $3 d$ and O $2 p$ spectral weights dominate. We also have shown how to reduce the La $5 p$ signal in HAXPES as to enhance relatively the Co $3 d$ by choosing the so-called perpendicular geometry. These results for $\mathrm{LaCoO}_{3}$ are representative for a wide range of rare-earth containing $3 d$ transition metal oxides: The interpretation of their HAXPES valence band spectra must consider the fact that the tiny contribution of the rare-earth $5 p$ to the valence band electronic structure is overcompensated by the extremely large photoionization cross section. We have further shown that the one-step photoemission method provides an excellent description of the HAXPES valence band spectra and their polarization dependence. Analysis on the basis of photoionization cross sections yields also highly satisfactory results, which is important since this requires less computing efforts than the one step calculations and is also applicable to evaluate strongly correlated systems for which band structure calculations are generally not adequate. Finally, we also show that an accurate quantitative description of the polarization dependence in the HAXPES spectra can be achieved if side-scattering effects are taken into consideration.

\section{ACKNOWLEDGMENTS}

We would like to thank T. Lorenz and C. Zobel from the University of Cologne for supplying us with the $\mathrm{Sr}$ doped $\mathrm{LaCoO}_{3}$ crystals. We acknowledge support from the Max Planck-POSTECH-Hsinchu Center for Complex Phase Materials. The research in Cologne was supported by the Deutsche Forschungsgemeinschaft (DFG) through SFB 608. J.M and L.N. would like to thank the CEDAMNF (CZ.02.1.01/0.0/0.0/15_003/0000358) cofunded by the Ministry of Education, Youth and Sports of Czech Republic. 
[1] J. C. Woicik (ed.), Hard X-ray Photoelectron Spectroscopy (HAXPES), Springer Series in Surface Sciences (Springer International Publishing, Cham, 2016).

[2] G. Panaccione, G. Cautero, M. Cautero, A. Fondacaro, M. Grioni, P. Lacovig, G. Monaco, F. Offi, G. Paolicelli, M. Sacchi, N. Stojić, G. Stefani, R. Tommasini, and P. Torelli, J. Phys.: Condens. Matter 17, 2671 (2005).

[3] A. Sekiyama, J. Yamaguchi, A. Higashiya, M. Obara, H. Sugiyama, M. Y. Kimura, S. Suga, S. Imada, I. Nekrasov, M. Yabashi, K. Tamasaku, and T. Ishikawa, New J. Phys. 12, 043045 (2010).

[4] S. Ouardi, G. H. Fecher, X. Kozina, G. Stryganyuk, B. Balke, C. Felser, E. Ikenaga, T. Sugiyama, N. Kawamura, M. Suzuki, and K. Kobayashi, Phys. Rev. Lett. 107, 036402 (2011).

[5] A. Sekiyama, A. Higashiya, and S. Imada, J. Electron Spectrosc. Relat. Phenom. 190, 201 (2013).

[6] S. Ouardi, G. H. Fecher, and C. Felser, J. Electron Spectrosc. Relat. Phenom. 190, 249 (2013).

[7] J. Weinen, T. C. Koethe, C. F. Chang, S. Agrestini, D. Kasinathan, Y. F. Liao, H. Fujiwara, C. Schüßler-Langeheine, F. Strigari, T. Haupricht, G. Panaccione, F. Offi, G. Monaco, S. Huotari, K.-D. Tsuei, and L. H. Tjeng, J. Electron Spectrosc. Relat. Phenom. 198, 6 (2015).

[8] A. Sekiyama, J. Electron Spectrosc. Relat. Phenom. 208, 100 (2016).

[9] A. K. Efimenko, N. Hollmann, K. Hoefer, J. Weinen, D. Takegami, K. K. Wolff, S. G. Altendorf, Z. Hu, A. D. Rata, A. C. Komarek, A. A. Nugroho, Y. F. Liao, K.-D. Tsuei, H. H. Hsieh, H.-J. Lin, C. T. Chen, L. H. Tjeng, and D. Kasinathan, Phys. Rev. B 96, 195112 (2017).

[10] S. Ueda and I. Hamada, J. Phys. Soc. Jpn. 86, 124706 (2017).

[11] Y. Utsumi, D. Kasinathan, K.-T. Ko, S. Agrestini, M. W. Haverkort, S. Wirth, Y.-H. Wu, K.-D. Tsuei, D.-J. Kim, Z. Fisk, A. Tanaka, P. Thalmeier, and L. H. Tjeng, Phys. Rev. B 96, 155130 (2017).

[12] K. Yamagami, Y. Kanai, S. Naimen, H. Fujiwara, T. Kiss, A. Tanaka, A. Higashiya, S. Imada, T. Kadono, K. Tamasaku, T. Muro, M. Yabashi, T. Ishikawa, H. Eisaki, S. Miyasaka, S. Tajima, and A. Sekiyama, Physica B: Condensed Matter 536, 843 (2018).
[13] N. Tsuda, K. Nasu, A. Yanase, and K. Siratori, Electronic Conduction in Oxides, Springer Series in Solid-State Sciences Vol. 94 (Springer, Berlin, 1991).

[14] M. Imada, A. Fujimori, and Y. Tokura, Rev. Mod. Phys. 70, 1039 (1998).

[15] J. J. Yeh and I. Lindau, At. Data Nucl. Data Tables 32, 1 (1985).

[16] M. B. Trzhaskovskaya, V. I. Nefedov, and V. G. Yarzhemsky, At. Data Nucl. Data Tables 77, 97 (2001).

[17] M. B. Trzhaskovskaya, V. I. Nefedov, and V. G. Yarzhemsky, At. Data Nucl. Data Tables 82, 257 (2002).

[18] M. B. Trzhaskovskaya, V. K. Nikulin, V. I. Nefedov, and V. G. Yarzhemsky, At. Data Nucl. Data Tables 92, 245 (2006).

[19] R. R. Heikes, R. C. Miller, and R. Mazelsky, Physica 30, 1600 (1964).

[20] G. Blasse, J. Appl. Phys. 36, 879 (1965).

[21] G. H. Jonker, J. Appl. Phys. 37, 1424 (1966).

[22] J. B. Goodenough and P. M. Raccah, J. Appl. Phys. 36, 1031 (1965).

[23] P. M. Raccah and J. B. Goodenough, Phys. Rev. 155, 932 (1967).

[24] J. Goodenough, Prog. Solid State Chem. 5, 145 (1971).

[25] M. W. Haverkort, Z. Hu, J. C. Cezar, T. Burnus, H. Hartmann, M. Reuther, C. Zobel, T. Lorenz, A. Tanaka, N. B. Brookes, H. H. Hsieh, H.-J. Lin, C. T. Chen, and L. H. Tjeng, Phys. Rev. Lett. 97, 176405 (2006).

[26] J. Braun, Rep. Prog. Phys. 59, 1267 (1996).

[27] J. Braun, J. Minár, and H. Ebert, Phys. Rep. 740, 1 (2018).

[28] H. Ebert, D. Ködderitzsch, and J. Minár, Rep. Prog. Phys. 74, 096501 (2011).

[29] M. Kriener, C. Zobel, A. Reichl, J. Baier, M. Cwik, K. Berggold, H. Kierspel, O. Zabara, A. Freimuth, and T. Lorenz, Phys. Rev. B 69, 094417 (2004).

[30] Y. Tokura, Y. Okimoto, S. Yamaguchi, H. Taniguchi, T. Kimura, and H. Takagi, Phys. Rev. B 58, R1699 (1998).

[31] T. Saitoh, T. Mizokawa, A. Fujimori, M. Abbate, Y. Takeda, and M. Takano, Phys. Rev. B 55, 4257 (1997).

[32] Using FPLO band structure calculations we determined that the La $p$ density of states in the valence band region originates primarily from the La $5 p$ semicore level. The contribution of the La $6 p$ is two orders of magnitude smaller. 